\title{
Kollaps oder Rückkehr zur Politik?
}

Die Rio+10-Konferenz nächstes Jahr in Johannesburg wirft ihre Schatten voraus. Nicht nur in Deutschland und anderen Ländern werden die diesbezüglichen Aktivitäten verstärkt, auch die Europäische Union plant, Ende Juni eine Nachhaltigkeitsstrategie zu beschließen. Im Mittelpunkt der Diskussion steht die Frage, wie der laufende Prozess zur Integration der Umweltpolitik in andere Politikbereiche mit der sozialen und ökonomischen Dimension der Nachhaltigkeit verknüpft werden kann bzw. sollte.

$\mathrm{D}$ Von Philipp Schepelmann ie Europäische Kommission wird im Juni dem Europäischen Rat in Göteborg die erste Nachhaltige Entwicklungsstrategie der EU vorlegen. Die Strategie wird zur Zeit von einer speziell zu diesem Zweck eingerichteten Einheit aus Beamten der verschiedenen betroffenen Generaldirektionen erstellt. Die Leitung dieser beim Generalsekretariat der Europäischen Kommission angesiedelten Arbeitseinheit wird von einer Gruppe von KommissarInnen wahrgenommen. Diese Gruppe zu ,Wachstum, Wettbewerbsfähigkeit, Beschäftigung und Nachhaltiger Entwicklung" trifft sich in unregelmäßigen Abständen unter der Leitung von Kommissionspräsident Romano Prodi. Der bisherige Entwurf einer Gliederung der Strategie sieht die Behandlung folgender zentraler Themen vor:

- soziale Ausgrenzung/Armut;

- öffentliche Gesundheit;

- demographische Entwicklung/Alterung der Gesellschaft;

- Klimawandel/saubere Energieformen;

- Erschöpfung natürlicher Ressourcen sowie

- Mobilität und Landnutzung.

Neben der Nachhaltigen Entwicklungsstrategie wird die Kommission auch ein Weißbuch zum Thema „Regieren“ (governance) präsentieren. Das Europäische Beratende Forum für Umwelt und Nachhaltige Entwicklung hat deshalb offensiv die Meinung vertreten, dass die Nachhaltigkeitsstrategie selber einen Testfall für gutes Regieren in Europa darstelle. In der Tat kämpft auch die Kommission, wie alle europäischen Regierungen, mit dem Problem der Politik-Kohärenz. Die Tatsache, dass sich unterschiedliche Regierungsebenen und -ressorts oft eher als Konkurrenz oder sogar als politische Gegner wahrnehmen, ist besonders bei der Querschnittsaufgabe Umweltschutz problematisch. In der Bundesrepublik kann man zur
Zeit die Schwierigkeit, kohärente und unterscheidbare Politikkonzepte durchzusetzen, im Bereich der Landwirtschaftspolitik studieren. Die von Erhard Eppler beschworene „,Rückkehr der Politik“ im Gegensatz zur „Kugellagerfunktion“ vieler Regierungsapparate für partikulare (Wirtschafts-)Interessen scheint eine zentrale Vorraussetzung für eine nachhaltige Entwicklung Europas zu sein.

\section{Der Cardiff-Prozess: Politikintegration als Programm}

Die Europäische Union hat im Vergleich zu den meisten europäischen Regierungen eine eindrucksvolle Programmatik zur Erreichung von mehr Politik-Kohärenz, worauf sich auch die Nachhaltige Entwicklungsstrategie stiutzen darf. Zum einen ist die Nachhaltigkeit mit allen drei Dimensionen (Ökologie, Wirtschaft und Soziales) im Artikel 2 des EG-Vertrages, der die Ziele der Gemeinschaft definiert, festgeschrieben. Die Politik-Kohärenz wird im Sinne einer nachhaltigen Entwicklung jedoch besonders prominent durch den Artikel 6 der Gemeinschaft gestïtzt. Dieser Artikel ist eine Querschnittsklausel des Amsterdamer Vertrages und sieht die Integration von Umweltbelangen in alle Politiken der Gemeinschaft vor.

Dieser Ausgabe beinhaltet eine Beilage der

UmweltFinanzGmbH

und der Zeitschrift

Politische Ökologie Nr. 67/68

„Aktie Grün - Investition in die Zukunft?"

Wir bitten unsere Leser um freundliche Beachtung.
Seit der Einführung des Artikel 6 im Jahr 1997 hat der Europäische Rat der Staats- und Regierungschefs einen komplexen Prozess zur Umweltintegration der Gemeinschaftspolitik initiiert (1). Im Sommer 1998 legte die Europäische Kommission in Cardiff ein Strategiepapier mit Leitlinien zur Durchfiuhrung der Integration vor. Der Rat forderte die relevanten Ratsformationen auf, in ihren jeweiligen Bereichen Strategien mit Zielen, Zeitrahmen, Indikatoren und Monitoringsystemen für die Umweltintegration zu schaffen. Dabei fassten die europäischen Staats- und Regierungschefs in Cardiff zunächst die umweltpolitisch besonders relevanten Sektoren Verkehr, Energie und Landwirtschaft ins Auge. Später folgten im Dezember 1998 in Wien die Sektoren Entwicklung, Binnenmarkt und Industrie und schließlich im Sommer 1999 in Köln die Sektoren Allgemeine Angelegenheiten, Wirtschaft und Finanzen sowie Fischerei. Alle betroffenen Ratsformationen wurden im Dezember 1999 in Helsinki gebeten, die Erarbeitung von Integrationsstrategien einschließlich Zeitplänen und Indikatoren bis zum Gipfel von Göteborg im Juni 2001 abzuschließen (2). Instrumente zur Evaluierung, Follow up und Monitoring sollen von der Kommission und dem Rat gemeinsam entwikkelt werden. Bemerkenswerterweise hat die deutsche Regierung kaum eigene Initiativen im Rahmen dieses für die Umweltpolitik Europas äußerst bedeutsamen Prozesses entwickelt.

\section{Der Impuls von Lissabon: Chance oder Risiko?}

Neben der Umweltintegration der Europäischen Union gibt es auch eine Integration der Wirtschafts- und Sozialpolitik. Nachdem die Wirtschafts- und Währungsunion weitgehend verwirklicht wird, ist die Sozialpolitik der Gemeinschaft noch stark unterentwickelt. Beide Politiken sind seit dem letztjährigen Europäischen Rat in Lissabon im Begriff, sich zu einer sozio-ökonomischen Gemeinschaftspolitik zu vereinigen. Dies finden unter anderem Ausdruck in den Strukturindikatoren der Gemeinschaft, die neuerdings die gemeinschaftlichen Ziele im Bereich Wirtschaftsund Sozialpolitik ausdrücken. Zwar findet in der Liste der Strukturindikatoren auch die Dimension nachhaltige Entwicklung ihren Niederschlag, es darf jedoch bezweifelt werden, dass der einzige dafür vorgesehene Indikator, nämlich Energieeffizienz, hier ausreicht.

Es mehren sich daher die Stimmen, die demgegenüber betonen, dass Nachhaltige Entwicklung nicht eine Umschreibung der Umweltdimension 
Neuerscheinung:

UnternehmensGrün Band 7

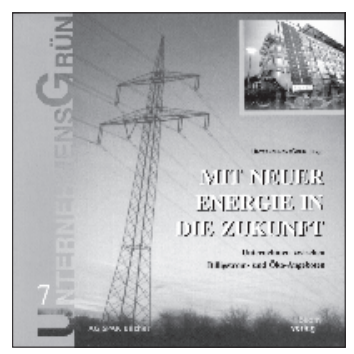

Mit neuer Energie in die Zukunft

Unternehmen zwischen Billigstrom und Öko-Angeboten

Seit einiger Zeit beginnt die Öffnung des Energiemarktes zu greifen: Große Energieversorgungsunternehmen buhlen mit Strom zu Tiefstpreisen um die KundInnen. Daneben präsentieren sich zahlreiche Newcome mit unterschiedlichen Ökostrom-Angeboten auf dem Markt. Wie können die Unternehmen als Verbraucher darauf reagieren? Band 7 der Reihe UnternehmensGrün informiert über die vielfältigen Chancen einer ökologischen und regenerativen Energiezu kunft. Fachleute aus Wissenschaft, Wirt schaft, Politik und Finanzwelt berichten über die neuesten Entwicklungen auf dem Energiemarkt und wie jeder davon profitieren kann.

Unternehmensgrün, Verband zur Förderung umweltgerechten Wirtschaftens (Hrsg.), Band 7, 168 Seiten, 25 DM, ökom Verlag, ISBN 3-925244-66-3.

Reihe UnternehmensGrün ist DIE Basislektüre für das Unternehmen der Zukunft: Band 1

"Augen zu und durch?

Was kommt nach der Krise?" - Über den konstruktiven Umgang mit der Rezession

Band 2

Erhalt durch Veränderung.

Perspektiven einer ökologischen und sozialen Umsteuerung

Band 3

Konkurrenten oder Partner.

Ökobilanz undf Öko-Audit im Verlgeich

Band 4

Von der Vision zur Praxis.

Nachhaltiges Wirtschaften als Perspektive für Unternehmen

Band 5

Märkte ohne Grenzen.

Unternehmen im Spannungsfeld zwischen nachhaltigen Wirtschaften und globalen Wettbewerb

Band 6

Arbeit - Strategien der Existenzsicherung

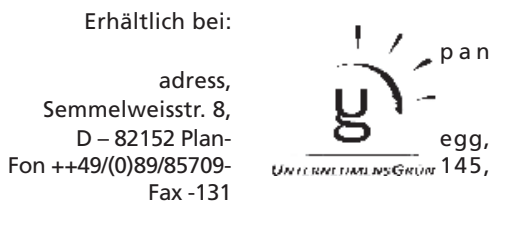

der Gemeinschaftspolitik sei, sondern ein Konzept, dass die Sozial-, Wirtschafts- und Umweltpolitik umfasse und miteinander verbände. Folgerichtig müsse die Nachhaltige Entwicklungsstrategie eine politische Klammer für den umweltpolitischen Cardiff- und den sozio-ökonomischen Lissabon-Prozess sein. Zwar wird zu recht die Frage aufgeworfen, ob denn so eine Mega-Strategie überhaupt noch planbar sei. Auf der anderen Seite droht sich jedoch der Lissabonner Hauptstrom von seinem kleinen Bruder, dem Cardiff-Prozess, abzukoppeln. Dies könnte die Folge haben, dass sich die Kluft zwischen gemeinschaftlicher Struktur- und Umweltpolitik weiter vertieft. Die bisher erreichten - vor allem symbolischen - Erfolge der Umweltintegration würden danach wieder zunichte gemacht. Es droht ein Rückfall der gemeinschaftlichen Umweltpolitik vom Status der Querschnittsaufgabe aller Ressorts zur isolierten Aufgabe eines machtlosen Juniorressorts.

Eine mangelnde Integration von Umweltbelangen in andere Ressorts ist bereits in der Evaluierung des Fünften EU-Umweltaktionsprogramms, das von 1992 bis 2000 lief, als Hauptgrund für das Scheitern der Umweltpolitik in Europa benannt worden. Ein Kollaps des ressortumfassenden Cardiff-Prozesses würde wahrscheinlich auch einen Misserfolg des Sechsten Umweltaktionsprogramms zur Folge haben. Das Aktionsprogramm, das im Februar von der Kommission im Entwurf vorgestellt wurde, geht jetzt in den Abstimmungsprozess mit dem Europäischen Parlament (3). Das im Vergleich zu seinem Vorgänger eher schwache und unverbindliche Programm wird neben der Nachhaltigkeitsstrategie und dem Cardiff-Prozess die Umweltpolitik in Europa in den nächsten Jahren maßgeblich bestimmen.

\section{> Offene Fragen}

Weiterhin offen ist die Frage, wie die Kommission bei der Erarbeitung und Umsetzung der Nachhaltigkeitsstrategie die Öffentlichkeit beteiligen wird. Nachhaltigkeit ist seit dem Beginn des Diskurses Anfange der 90er Jahre stark mit der Beteiligung verschiedenster Interessensgruppen assoziiert. Zudem wäre eine umfassende Nachhaltigkeitsstrategie wertlos, wenn sich die verschiedenen Akteure auf staatlicher, nicht-staatlicher, europäischer und nationaler Ebene nicht mit der Strategie identifizieren könnten.

Mit dieser Vorstellung einer abgehobenen Strategie aus dem Elfenbeinturm der Brüsseler Bürokratie verbindet sich schließlich auch das worst-
case-Szenario: Die Kommission legt in Göteborg irgendein Papier vor, mit dem sich niemand identifizieren kann, das aber gut genug ist, um gemeinschaftliche Aktivität im Hinblick auf die Rio+10-Konferenz in Johannesburg im September 2002 belegen zu können. Dieses bedeutungslose Papier würde den Cardiff-Prozess ablösen und weitgehend losgelöst von Lissabon und dem Sechsten Umweltaktionsprogramm im Raum stehen und dann irgendwann in einer Schublade mangels Interesse des Präsidenten der Europäischen Kommission verschwinden (4).

\section{Anmerkungen}

(1) Vgl. zum Umweltintegrationsprozess auch die Studien des Wuppertal-Instituts "Von Wien nach Helsinki. Umweltpolitische Anforderungen an den Prozess zur Integration von Umweltbelangen in andere Politikbereiche der Europäischen Union"; Download unter http://www.wupperinst.org/Publikationen/EU-Policy-Papers/ sowie des Sustainable Europe Research Institutes „Von Helsinki nach Göteborg. Evaluierung der Umweltintegration in der Europäischen Union". Download unter http://www.seri.at/europa/abgeschlossen/umweltintegration.htm

(2) Vgl. hierzu z.B. die aktuelle Studie von SPRU und Wuppertal-Institut "Indicators for Monitoring Integration of Environment and Sustainable Development in Enterprise Policy". Download unter http://www.sussex.ac.uk/spru/environment/research/indicator/intind.html

(3) Nähere Informationen zum Sechsten Umweltaktionsprogramm sowie dem weiteren Abstimmungsprozess finden sich im Internet unter http://www.europa.eu.int/comm/ environment/newprg

(4) Um diesem Größten Anzunehmenden Unfall der europäischen Umweltpolitik vorzubeugen, haben die Regierungen von Österreich, Deutschland und Großbritannien zusammen mit der Europäischen Kommission und der Schwedischen Präsidentschaft Anfang April eine Konferenz zum Thema veranstaltet. Nähere Informationen bei Frau Rita Trattnig, Österreichisches Umweltministerium, E-mail: rita.trattnig@ bmu.gv.at.

\section{Der Autor}

Philipp Schepelmann ist Leiter des Projektbereiches Umweltintegration am Wuppertal Institut für Klima, Umwelt und Energie und des Projektbereiches Europa am Sustainable Europe Research Institut (SERI). Kontakt: Birresborner Str.24, 50935 Köln, E-mail: Philipp.Schepelmann@t-online.de 
(c) 20I0 Authors; licensee IÖW and oekom verlag. This is an article distributed under the terms of the Creative Commons Attribution Non-Commercial No Derivates License (http://creativecommons.org/licenses/by-nc-nd/3.o/), which permits unrestricted use, distribution, and reproduction in any medium, provided the original work is properly cited. 